\title{
A Realization of Hom-Lie Algebras by Iso-Deformed Commutator Bracket
}

\author{
Xiuxian Li \\ Department of Mathematics, Tongji University, Shanghai 200092, China \\ Correspondence should be addressed to Xiuxian Li; lxxcaptain@126.com
}

Received 21 March 2013; Accepted 19 June 2013

Academic Editor: Shi Weichen

Copyright (C) 2013 Xiuxian Li. This is an open access article distributed under the Creative Commons Attribution License, which permits unrestricted use, distribution, and reproduction in any medium, provided the original work is properly cited.

We construct classical Iso-Lie and Iso-Hom-Lie algebras in $g l(V)$ by twisted commutator bracket through Iso-deformation. We prove that they are simple. Their Iso-automorphisms and isotopies are also presented.

\section{Introduction}

The conventional Lie theory has been developed in mathematical literature in its linear, local, and canonical formulation and the simplest conceivable product $[A, B]=A B-$ $B A$, where $A B$ is the trivial associative product. But it is not applicable to a growing number of nonlinear, nonlocal, and noncanonical systems which have recently emerged in mathematics. Lie-isotopic theory [1-3] is the generalization of the unit $I$ to a new one $\widehat{I}$ called isotopic unit or isounit. The resulting new mathematical structures include the old ones as special cases.

Hom-Lie algebras $(\mathfrak{g},[\cdot, \cdot], \alpha)$ were first introduced by Hartwig et al. in [4] to describe the structure of deformation of the Witt and Virasoro algebra in 2006. They are a generalization of Lie algebras. When $\alpha=i d$, the Hom-Lie algebras degenerate to exactly the Lie algebras. Because Hom-Lie algebras are closely related to discrete and deformed vector fields, differential calculus $[5,6]$, they have been researched extensively these years [7-9].

An elementary but important property of Lie algebras is that each associative algebra gives rise to a Lie algebra via the commutator bracket. But it is not natural to define Hom-Lie algebras by this way. In [10], Makhlouf and Silvestrov introduced the notion of a Hom-associative algebra $(\mathfrak{g}, \mu, \alpha)$, in which the binary operation $\mu$ satisfies an $\alpha$ twisted version of associativity. The authors mentioned that Lie-Santilli isotopies [1-3] can be modified appropriately to suit the Hom-algebras context. The relations between Hom-Lie algebras and Santilli's deformed bracket products are certainly interesting. This method solves the question how can commutator products in a linear space be modified to yield as general as possible (Hom-)Lie algebras.

The structure of Lie algebras over $\mathbb{C}$ is better understood now [11, 12]. Classical simple Lie algebras over $\mathbb{C}$ can be classified into $A, B, C, D$ types. Similarly, we can use the twisted commutator bracket through Iso-deformation to study classical Iso-(Hom-)Lie algebras over $\mathbb{C}$.

The paper is organized as follows: in Section 2, we study relations between multiplicative Hom-Lie algebras and Lie algebras; in Section 3, we construct Iso-Lie and Iso-HomLie algebras; in Section 4, classical II types $A, B, C, D$-IsoLie and Iso-Hom-Lie algebras are studied. We prove that they are simple. Their Iso-automorphism and isotopy are also presented.

Throughout this paper, we denote by " $E$ " the identity matrix, " $g L(V)$ " the linear group, and " $x$ " the transpose

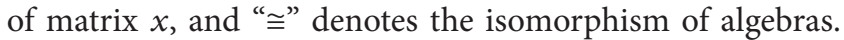
Without otherwise stated, all algebras are finite dimensional and over the complex field $\mathbb{C}$. 


\section{Hom-Lie Algebras}

Definition 1 (see [4]). A Hom-Lie algebra is a triple $(\mathfrak{g},[\cdot, \cdot], \alpha)$ consisting of a vector space $\mathfrak{g}$ over $\mathbb{C}$, a linear sef-map $\alpha$, and a bilinear map $[\cdot, \cdot]: \mathfrak{g} \times \mathfrak{g} \rightarrow \mathfrak{g}$ satisfying

$$
\begin{array}{r}
{[x, y]=-[y, x], \quad \forall x, y \in \mathfrak{g} ;} \\
{[\alpha(x),[y, z]]+[\alpha(y),[z, x]]+[\alpha(z),[x, y]]=0,} \\
\forall x, y, z \in \mathfrak{g} .
\end{array}
$$

When $\alpha=i d,(\mathfrak{g},[\cdot, \cdot], \alpha)$ is degenerated to Lie algebra $(\mathfrak{g},[\cdot, \cdot])$. A Hom-Lie algebra $(\mathfrak{g},[\cdot, \cdot], \alpha)$ is called multiplicative if

$$
\alpha([x, y])=[\alpha(x), \alpha(y)], \quad \forall x, y \in \mathfrak{g}
$$

is satisfied [13]. We call $(\mathfrak{g},[\cdot, \cdot], \alpha)$ Lie-type if there exists a Lie algebra $\left(\mathfrak{g},[\cdot, \cdot]^{\prime}\right)$ such that $[x, y]=\alpha\left([x, y]^{\prime}\right)=$ $[\alpha(x), \alpha(y)]^{\prime}, \forall x, y \in \mathfrak{g}$. At the same time $\left(\mathfrak{g},[\cdot, \cdot]^{\prime}\right)$ is called the Hom-Lie admissible algebra of $(\mathfrak{g},[\cdot, \cdot], \alpha)$.

A subspace $\mathfrak{g}_{1}$ of $\mathfrak{g}$ is called an ideal of $(\mathfrak{g},[\cdot, \cdot], \alpha)$ if $\alpha\left(\mathfrak{g}_{1}\right) \subseteq$ $\mathfrak{g}_{1},\left[\mathfrak{g}_{1}, \mathfrak{g}\right] \subseteq \mathfrak{g}_{1}$ are satisfied. We call a Hom-Lie algebra $(\mathfrak{g},[\cdot, \cdot], \alpha)$ simple if it has no nontrivial ideals and $[\mathfrak{g}, \mathfrak{g}]=\mathfrak{g}$.

An automorphism $\psi$ of a Hom-Lie algebra $(\mathfrak{g},[\cdot, \cdot], \alpha)$ is an invertible linear self-map satisfying $\psi([x, y])=$ $[\psi(x), \psi(y)], \forall x, y \in \mathfrak{g}$ and $\psi \alpha=\alpha \psi$.

An isomorphism of Hom-Lie algebras $\left(\mathfrak{g}_{1},[\cdot, \cdot]_{1}, \alpha_{1}\right)$ with $\left(\mathfrak{g}_{2},[\cdot, \cdot]_{2}, \alpha_{2}\right)$ is an invertible linear map $\phi: \mathfrak{g}_{1} \rightarrow \mathfrak{g}_{2}$ satisfying $\phi\left([x, y]_{1}\right)=[\phi(x), \phi(y)]_{2}, \forall x, y \in \mathfrak{g}_{1}$ and $\alpha_{2} \phi=$ $\phi \alpha_{1}$.

Lemma 2. Let $(\mathfrak{g},[\cdot, \cdot], \alpha)$ be a multiplicative simple Hom-Lie algebra. Then $\alpha$ is invertible.

Proof. It is easy to check that $\operatorname{Ker}(\alpha)$ is an ideal of $(\mathfrak{g},[\cdot, \cdot], \alpha)$. By the simplicity of $(\mathfrak{g},[\cdot, \cdot], \alpha)$, we have $\operatorname{Ker}(\alpha)=0$. That is, $\alpha$ is invertible.

Lemma 3 (see [14]). Let $(\mathfrak{g},[\cdot, \cdot])$ be a Lie algebra over $\mathbb{C}$ with an algebraic homomorphism $\alpha$. Define a bracket $[\cdot, \cdot]_{\alpha}$ by $[x, y]_{\alpha}=\alpha([x, y]), \forall x, y \in \mathfrak{g}$. Then $\left(\mathfrak{g},[\cdot, \cdot]_{\alpha}, \alpha\right)$ is a Hom-Lie algebra.

Theorem 4. Let $(\mathfrak{g},[\cdot, \cdot], \alpha)$ be a multiplicative Hom-Lie algebra with $\alpha$ invertible. Then $(\mathfrak{g},[\cdot, \cdot], \alpha)$ is Lie-type with the Hom-Lie admissible algebra $\left(\mathfrak{g},[\cdot, \cdot]^{\prime}\right)$, where $[\cdot, \cdot]^{\prime}$ is defined by $[x, y]^{\prime}=\alpha^{-1}([x, y]), \forall x, y \in \mathfrak{g}$.

Proof. Define $[x, y]^{\prime}=\alpha^{-1}([x, y]), \forall x, y \in \mathfrak{g}$. It is easy to check that

$$
\alpha^{-1}\left([x, y]^{\prime}\right)=\left[\alpha^{-1}(x), \alpha^{-1}(y)\right]^{\prime}, \quad \forall x, y \in \mathfrak{g} .
$$

Now we prove that $\left(\mathfrak{g},[\cdot, \cdot]^{\prime}\right)$ is a Lie algebra. The skewsymmetricity of $[\cdot, \cdot]^{\prime}$ is obvious. $\forall x, y, z \in \mathfrak{g}$,

$$
\begin{aligned}
\mho_{x, y, z}\left[x,[y, z]^{\prime}\right]^{\prime} & =\mho_{x, y, z} \alpha^{-1}\left(\left[x, \alpha^{-1}([y, z])\right]\right) \\
& =\mho_{x, y, z} \alpha^{-1}\left(\left[\alpha^{-1} \alpha(x), \alpha^{-1}([y, z])\right]\right) \\
& =\circlearrowleft_{x, y, z} \alpha^{-2}([\alpha(x),[y, z]])=0,
\end{aligned}
$$

here $\sigma_{x, y, z}$ denotes the cyclic summation over $x, y, z$. We have the conclusion.

Lemma 5 (see $[15])$. Let $\left(\mathfrak{g}_{1},[\cdot, \cdot]_{1}, \alpha\right)$ and $\left(\mathfrak{g}_{1},[\cdot, \cdot]_{2}, \beta\right)$ be Lie type Hom-Lie algebra with $\beta$ invertible. Then an invertible linear map $\phi$ is an isomorphism of Hom-Lie algebras if and only if $\phi$ is an isomorphism of their Hom-Lie admissible algebras satisfying $\beta \phi=\phi \alpha$.

Lemma 6 (see [15]). Let $(\mathfrak{g},[\cdot, \cdot], \alpha)$ be a Lie type Hom-Lie algebra with $\alpha$ invertible. Then $\psi$ is an automorphism of $(\mathfrak{g},[\cdot, \cdot], \alpha)$ if and only if $\psi$ is an automorphism of the Hom-Lie admissible algebra satisfying $\alpha \psi=\psi \alpha$.

Proposition 7. Let $(\mathfrak{g},[\cdot, \cdot], \alpha)$ be a finite dimensional Lie type Hom-Lie algebra with $\alpha$ invertible. If its Hom-Lie admissible algebra is simple, then $(\mathfrak{g},[\cdot, \cdot], \alpha)$ is simple.

Proof. By Theorem 4, the Hom-Lie admissible algebra can be written as $\left(\mathfrak{g},[\cdot, \cdot]^{\prime}\right)$, where $[\cdot, \cdot]^{\prime}$ is defined by $[x, y]^{\prime}=$ $\alpha^{-1}([x, y]), \forall x, y \in \mathfrak{g}$. Suppose $[\mathfrak{g}, \mathfrak{g}] \varsubsetneqq \mathfrak{g}$, then

$$
[\mathfrak{g}, \mathfrak{g}]^{\prime}=\alpha^{-1}([\mathfrak{g}, \mathfrak{g}])=\left[\alpha^{-1}(\mathfrak{g}), \alpha^{-1}(\mathfrak{g})\right] \subseteq[\mathfrak{g}, \mathfrak{g}] \varsubsetneqq \mathfrak{g},
$$

which is a contradiction with the simplicity of $\left(\mathfrak{g},[\cdot, \cdot]^{\prime}\right)$. This reduces to $[\mathfrak{g}, \mathfrak{g}]=\mathfrak{g}$.

Let $\mathfrak{g}_{1}$ be a nontrivial ideal of $(\mathfrak{g},[\cdot, \cdot], \alpha)$. By definition there are $\alpha\left(\mathfrak{g}_{1}\right) \subseteq \mathfrak{g}_{1} ;\left[\mathfrak{g}_{1}, \mathfrak{g}\right] \subseteq \mathfrak{g}_{1}$. Therefore, $\left[\mathfrak{g}_{1}, \mathfrak{g}\right]^{\prime}=$ $\alpha^{-1}\left(\left[\mathfrak{g}_{1}, \mathfrak{g}\right]\right) \subseteq \alpha^{-1}\left(\mathfrak{g}_{1}\right) \subseteq \mathfrak{g}_{1}$. That is, $\mathfrak{g}_{1}$ is a nontrivial ideal of the Hom-Lie admissible algebra, which is impossible. We have the conclusion.

\section{Iso Algebras}

Let $\mathfrak{g}$ be an associative algebra with the conventional associative product $x y, \forall x, y \in \mathfrak{g}$ over $\mathbb{C}$. An Iso-associative algebra $(\mathfrak{g}, *)$ is the same vector space $\mathfrak{g}$ over $\mathbb{C}$ with a product * satisfying $(x * y) * z=x *(y * z), \forall x, y, z \in \mathfrak{g}$, where the product $*$ is defined by one of the following cases, the parameters $T, w$ appearing in cases II-V are fixed not necessary belonging to $\mathfrak{g}$ (it deserves to mention that in $[3],(\mathfrak{g}, *)$ is an Iso-associative algebra over an Iso-field $\widehat{\mathbb{C}}$, specially, in this paper we study Iso-algebra over $\mathbb{C}$ ):

$\mathrm{I}: x * y=a x y, 0 \neq a \in \mathbb{C}$ is fixed;

II: $x * y=x T y, \forall x, y \in \mathfrak{g}, T$ is invertible;

III: $x * y=w x w y w, \forall x, y \in \mathfrak{g}, w^{2}=w \neq 0$;

IV: $x * y=w x w T w y w, \forall x, y \in \mathfrak{g}, w^{2}=w, w, T \neq 0$; 
$\mathrm{V}: x * y=a w x w T w y w, x, y \in \mathfrak{g}, a \in \mathbb{C}, w^{2}=$ $w ; a, w, T \neq 0$.

It is obvious that of the above Iso-associative algebras only the first four are independent and there is no essential difference between type I and the conventional associative algebra.

Definition 8. An Iso-Lie algebra $(\mathfrak{g},[\cdot, \cdot])$ is a vector space $\mathfrak{g}$ over $\mathbb{C}$ with a bracket $[\cdot, \cdot]$ defined by $[x, y]=x * y-y *$ $x, \forall x, y \in \mathfrak{g}$, where $(\mathfrak{g}, *)$ is an Iso-associative algebra. At the same time $(\mathfrak{g}, *)$ is called the Lie admissible algebra of $(\mathfrak{g},[\cdot, \cdot])$.

We call an isomorphism of Iso-algebras an isotopy. An automorphism of an Iso-algebra is called an Isoautomorphism.

We call $(\mathfrak{g},[\cdot, \cdot], \alpha)$ an Iso-Hom-Lie algebra if its Hom-Lie admissible algebra is an Iso-Lie algebra.

Corollary 9. Let $(\mathfrak{g},[\cdot, \cdot])$ be an Iso-Lie algebra with an Iso-automorphism $\alpha$. Define a bracket $[\cdot, \cdot]_{\alpha}$ by $[x, y]_{\alpha}=$ $\alpha([x, y]), \forall x, y \in \mathfrak{g}$. Then $\left(\mathfrak{g},[\cdot, \cdot]_{\alpha}, \alpha\right)$ is an Iso-Hom-Lie algebra.

We call $(\mathfrak{g},[\cdot, \cdot])$ I-V type Iso-Lie algebras if their Lie admissible algebras are I-V type Iso-associative algebras, respectively. And call the Iso-Hom-Lie algebras constructed in Corollary $9 \mathrm{I}-\mathrm{V}$ type Iso-Hom-Lie algebras if their HomLie admissible algebras are I-V type Iso-Lie algebras, respectively. In this paper we put emphasis on type II Iso-(Hom-)Lie algebras.

\section{Classical Type II Iso-(Hom-)Lie Algebras}

In this section we study classical types II $A_{n}, B_{l}, C_{n}, D_{l}$-Iso(Hom-)Lie algebras over $\mathbb{C}$. Give their Iso-automorphisms and isotopies. Prove that they are simple.

Let $V$ be a finite vector space, $g l(V)$ is the linear space with the conventional associative product $x y, \forall x, y \in g l(V)$. $T \in g l(V)$ is an invertible element, $\mathfrak{g}_{T} \subseteq \operatorname{gl}(V) .\left(\mathfrak{g}_{T},[\cdot, \cdot]_{T}\right)$ is an II type Iso-Lie algebra over $\mathbb{C}$, with the bracket defined by $[x, y]_{T}=x T y-y T x, \forall x, y \in \mathfrak{g}_{T}$. It is easy to know that the conventional linear Lie algebra over $\mathbb{C}$ is a special II type Iso-Lie algebra when $T$ is degenerated to the trivial identity.

Theorem 10. Let $s l(\widehat{n+1, \mathbb{C}})_{T}$ be a vector space satisfying

$$
s l(\widehat{n+1, \mathbb{C}})_{T}=\{\hat{x} \in g l(V) \mid \operatorname{Tr}(T \hat{x})=0\} .
$$

(1) Define a bracket $[\cdot, \cdot]_{T}$ by $[\widehat{x}, \widehat{y}]_{T}=\widehat{x} T \widehat{y}-\widehat{y} T \widehat{x}, \forall \hat{x}, \widehat{y} \in$ $\operatorname{sl}(\widehat{n+1, \mathbb{C}})_{T}$. Then $\left(\operatorname{sl}(\widehat{n+1, \mathbb{C}})_{T},[\cdot, \cdot]_{T}\right)$ is an II type Iso-Lie algebra (one calls it an $A_{n}$-Iso-Lie algebra).

(2) Define an invertible linear map $\alpha_{P}$ by

$$
\alpha_{P}(\widehat{x})=P^{-1} \widehat{x} P, \quad \forall \hat{x} \in \operatorname{sl}(\overline{n+1, \mathbb{C}})_{T},
$$

where $P \in g L(V)$ satisfying $P T=T P$, and then $\alpha_{P}$ is an Iso-automorphism of $\left(\operatorname{sl}(\widehat{n+1, \mathbb{C}})_{T},[\cdot, \cdot]_{T}\right)$.
(3) There is an isotopy between the conventional $A_{n}$-type Lie algebra $(\operatorname{sl}(n+1, \mathbb{C}),[\cdot, \cdot])$ and $\left(\operatorname{sl}(\widehat{n+1, \mathbb{C}})_{T},[\cdot, \cdot]_{T}\right)$. Moreover, $\left(\operatorname{sl}(\widehat{n+1, \mathbb{C}})_{T},[\cdot, \cdot]_{T}\right)$ is simple.

(4) Let $\left(\operatorname{sl}(\widehat{n+1, \mathbb{C}})_{T_{1}},[\cdot, \cdot]_{T_{1}}\right)$ and $\left(\operatorname{sl}(\widehat{n+1, \mathbb{C}})_{T_{2}},[\cdot, \cdot]_{T_{2}}\right)$ be $A_{n}$-Iso-Lie algebras, and then they are isotopic.

(5) Define a new bracket $[\cdot, \cdot]_{T \alpha_{P}}$ by

$$
\begin{aligned}
\left([\hat{x}, \hat{y}]_{T \alpha_{P}}\right)=\alpha_{P}\left([\hat{x}, \hat{y}]_{T}\right)= & P^{-1}(\hat{x} T \hat{y}-\widehat{y} T \hat{x}) P, \\
& \forall \hat{x}, \hat{y} \in \operatorname{sl}(\widehat{n+1, \mathbb{C}})_{T} .
\end{aligned}
$$

Then $\left(\operatorname{sl}(\widehat{n+1, \mathbb{C}})_{T},[\cdot, \cdot]_{T \alpha_{P}}, \alpha_{P}\right)$ is a simple Iso-HomLie algebra (one calls it an $A_{n}$-Iso-Hom-Lie algebra) with an Iso-automorphism $\psi$ defined by $\psi(\widehat{x})=$ $Q^{-1} \widehat{x} Q, \forall \widehat{x} \in \operatorname{sl}(\widehat{n+1, \mathbb{C}})_{T}$, where $Q \in g L(V)$ satisfying $T Q=Q T$ and $P Q=k Q P$, for some $k \in \mathbb{C}$.

(6) Let $\left(\operatorname{sl}(n+1, \mathbb{C}),[\cdot, \cdot]_{\alpha_{Q}}, \alpha_{\mathrm{Q}}\right)$ be a Hom-Lie algebra with

$$
\begin{array}{r}
\alpha_{\mathrm{Q}}(x)=Q^{-1} x \mathrm{Q} ; \quad[x, y]_{\alpha_{Q}}=Q^{-1}(x y-y x) Q, \\
\forall x, y \in \operatorname{sl}(n+1, \mathbb{C}) .
\end{array}
$$

$$
\begin{aligned}
& \text { If } Q=k P, \text { for some } k \in \mathbb{C} \text { is satisfied, } \\
& \text { then }\left(\operatorname{sl}(n+1, \mathbb{C}),[\cdot, \cdot]_{\alpha_{Q}}, \alpha_{Q}\right) \text { is isotopic to } \\
& \left(\operatorname{sl}(\widehat{n+1, \mathbb{C}})_{T},[\cdot, \cdot]_{T \alpha_{P}}, \alpha_{P}\right) .
\end{aligned}
$$

(7) Let $\left(\operatorname{sl}(\widehat{n+1, \mathbb{C}})_{T_{1}},[\cdot, \cdot]_{T_{1} \alpha_{P}}, \alpha_{P}\right)$ and $\left(\operatorname{sl}(\widehat{n+1, \mathbb{C}})_{T_{2}}\right.$, $\left.[\cdot, \cdot]_{T_{2} \alpha_{Q}}, \alpha_{Q}\right)$ be $A_{n}$-Iso-Hom-Lie algebras. They are isotopic if and only if $P$ is conjugate with $k Q$ (for some $k \in \mathbb{C})$.

Proof. (1) $\forall \hat{x}, \widehat{y} \in \operatorname{sl}(\widehat{n+1, \mathbb{C}})_{T}$, there are $\operatorname{Tr}(T \hat{x})=0$; $\operatorname{Tr}(T \hat{y})=0$, then $\operatorname{Tr}\left(T[\hat{x}, \hat{y}]_{T}\right)=\operatorname{Tr}(T \hat{x} T \hat{y}-T \widehat{y} T \widehat{x})=0$; therefore, $[\hat{x}, \hat{y}]_{T} \in \operatorname{sl}\left(\widehat{n+1, \mathbb{C})_{T}}\right.$. The Jacobi identity can be checked directly. By Definition $8,\left(\operatorname{sl}(\widehat{n+1, \mathbb{C}})_{T},[\cdot, \cdot]_{T}\right)$ is an II-type Iso-Lie algebra.

(2) Because $T P=P T$, so $T P^{-1}=P^{-1} T$ and $P^{-1} T P=T$ are satisfied. Consider $\forall \widehat{x} \in s l(\widehat{n+1, \mathbb{C}})_{T}$,

$$
\begin{aligned}
\operatorname{Tr}\left(T \alpha_{P}(\widehat{x})\right) & =\operatorname{Tr}\left(T P^{-1} \widehat{x} P\right)=\operatorname{Tr}\left(P^{-1} T \widehat{x} P\right) \\
& =\operatorname{Tr}(T \widehat{x})=0 .
\end{aligned}
$$

Therefore $\alpha_{P}\left(\operatorname{sl}(\widehat{n+1, \mathbb{C}})_{T}\right) \subseteq \operatorname{sl}(\widehat{n+1, \mathbb{C}})_{T} \cdot \forall \hat{x}, \hat{y} \quad \in$ $\operatorname{sl}(\widehat{n+1, \mathbb{C}})_{T}$,

$$
\begin{aligned}
\alpha_{P}\left([\widehat{x}, \widehat{y}]_{T}\right) & =P^{-1}(\widehat{x} T \widehat{y}-\widehat{y} T \widehat{x}) P \\
& =P^{-1} \widehat{x} P P^{-1} T P P^{-1} \widehat{y} P-P^{-1} \widehat{y} P P^{-1} T P P^{-1} \widehat{x} P \\
& =P^{-1} \widehat{x} P T P^{-1} \widehat{y} P-P^{-1} \widehat{y} P T P^{-1} \widehat{x} P \\
& =\left[\alpha_{P}(\widehat{x}), \alpha_{P}(\widehat{y})\right]_{T} .
\end{aligned}
$$

So $\alpha_{P}$ is an Iso-automorphism of $\left(\operatorname{sl}(\widehat{n+1, \mathbb{C}})_{T},[\cdot, \cdot]_{T}\right)$. 
(3) Define an invertible linear map $\phi$ by

$$
\phi(x)=T^{-1} x, \quad \forall x \in \operatorname{sl}(n+1, \mathbb{C}) .
$$

It is obvious that $\phi$ is invertible and $\operatorname{Tr}(\operatorname{T} \phi(x))=0$; therefore, $\phi(s l(n+1, \mathbb{C})) \subseteq \operatorname{sl}(\widehat{n+1, \mathbb{C}})_{T}$.

$$
\begin{array}{r}
{[\phi(x), \phi(y)]_{T}=\phi(x) T \phi(y)-\phi(y) T \phi(x)} \\
=T^{-1} x T T^{-1} y-T^{-1} y T T^{-1} x \\
=T^{-1}(x y-y x)=\phi([x, y]), \\
\forall x, y \in \operatorname{sl}(n+1, \mathbb{C}) .
\end{array}
$$

We have the first conclusion.

Suppose $\widehat{\mathfrak{g}}_{T}$ is a nontrivial ideal of $\left(s l(\widehat{n+1, \mathbb{C}})_{T},[\cdot, \cdot]_{T}\right)$. Let $\mathfrak{g}=T \widehat{\mathfrak{g}}_{T}=\phi^{-1}\left(\widehat{\mathfrak{g}}_{T}\right)$. Then

$$
\begin{aligned}
{[\mathfrak{g}, s l(n+1, \mathbb{C})] } & =\phi^{-1}[\phi(\mathfrak{g}), \phi(s l(n+1, \mathbb{C}))] \\
& \subseteq \phi^{-1}\left(\left[\widehat{\mathfrak{g}}_{T}, s l(\widehat{n+1, \mathbb{C}})_{T}\right]_{T}\right) \\
& \subseteq \phi^{-1}\left(\widehat{\mathfrak{g}}_{T}\right)=\mathfrak{g} .
\end{aligned}
$$

Therefore, $\mathfrak{g}$ is a nontrivial ideal of $(\operatorname{sl}(n+1, \mathbb{C}),[\cdot, \cdot])$, which is a contradiction with the simplicity of $(\operatorname{sl}(n+1, \mathbb{C}),[\cdot, \cdot])$. We have that $\left(\operatorname{sl}(\widehat{n+1, \mathbb{C}})_{T},[\cdot, \cdot]_{T}\right)$ is simple.

(4) Define an invertible linear map $\phi$ by

$$
\phi(\widehat{x})=\widehat{x} T_{1} T_{2}^{-1}, \quad \forall \widehat{x} \in \operatorname{sl}(\widehat{n+1, \mathbb{C}})_{T_{1}} ;
$$

then

$$
\begin{aligned}
& \phi(\widehat{x}) \in \operatorname{sl}(\widehat{n+1, \mathbb{C}})_{T_{2}}, \\
& \phi\left([\hat{x}, \widehat{y}]_{T_{1}}\right)=[\phi(\widehat{x}), \phi(\widehat{y})]_{T_{2}}, \\
& \forall \hat{x}, \hat{y} \in \operatorname{sl}(\widehat{n+1, \mathbb{C}})_{T_{1}}
\end{aligned}
$$

can be checked as in (3) of the theorem. We have the conclusion.

(5) According to (2) and (3) of the theorem, Lemma 3, Proposition 7, and the definition of Iso-Hom-Lie algebra, we have that $\left(\operatorname{sl}(\widehat{n+1, \mathbb{C}})_{T},[\cdot, \cdot]_{T \alpha_{P}}, \alpha_{P}\right)$ is an simple Iso-Hom-Lie algebra. If $\psi$ is an Iso-automorphism of $\left(s l(\widehat{n+1, \mathbb{C}})_{T},[\cdot, \cdot]_{T \alpha_{P}}, \alpha_{P}\right)$, by Lemma $6, \psi$ is an Iso-automorphism of the Hom-Lie admissible algebra $\left(s l(\widehat{n+1, \mathbb{C}})_{T},[\cdot, \cdot]_{T}\right)$ and satisfying

$$
\psi \alpha_{P}=\alpha_{P} \psi
$$

By (2) of the theorem again we have $\psi(\widehat{x})=Q^{-1} \widehat{x} Q, \forall \widehat{x} \in$ $\operatorname{sl}(\widehat{n+1, \mathbb{C}})_{T}$, where $Q \in g L(V)$ satisfies $Q T=T Q$. Consider $\forall \widehat{x} \in \operatorname{sl}(\widehat{n+1, \mathbb{C}})_{T}$, and (17) is equivalent to

$$
\begin{aligned}
\psi \alpha_{P}(\widehat{x}) & =\alpha_{P} \psi(\widehat{x}) \\
\Longrightarrow Q^{-1} P^{-1} \widehat{x} P Q & =P^{-1} Q^{-1} \widehat{x} Q P \\
\Longrightarrow Q P Q^{-1} P^{-1} \widehat{x} & =\widehat{x} Q P Q^{-1} P^{-1}, \\
\forall \hat{x} & \in \operatorname{sl}(n \widehat{+1, \mathbb{C}})_{T} .
\end{aligned}
$$

By (3) of the proof, there exists $x^{\prime} \in \operatorname{sl}(n+1, \mathbb{C})$ such that $\widehat{x}=T^{-1} x^{\prime}$. Equation (18) is equivalent to

$$
\begin{aligned}
\mathrm{QPQ}^{-1} P^{-1} T^{-1} x^{\prime} & =T^{-1} x^{\prime} \mathrm{QPQ} \mathrm{Q}^{-1} P^{-1}=T^{-1} \mathrm{Q} P \mathrm{Q}^{-1} P^{-1} x^{\prime} \\
& \Longrightarrow x^{\prime} \mathrm{QPQ} \mathrm{Q}^{-1} P^{-1}=\mathrm{QPQ} \mathrm{Q}^{-1} P^{-1} x^{\prime}
\end{aligned}
$$

By the arbitrariness of $\widehat{x}$, we have for all $x^{\prime} \in \operatorname{sl}(n+1, \mathbb{C}),(19)$ is established. According to Schur's lemma we have $Q P=k P Q$, for some $k \in \mathbb{C}$.

(6) If $\phi$ is an isotopy from $\left(\operatorname{sl}(n+1, \mathbb{C}),[\cdot, \cdot]_{\alpha_{Q}}, \alpha_{\mathrm{Q}}\right)$ to $\left(\operatorname{sl}(n \widehat{+1, \mathbb{C}})_{T},[\cdot, \cdot]_{T \alpha_{P}}, \alpha_{P}\right)$; according to Lemma $5, \phi$ is an isotopy of their Hom-Lie admissible algebras and satisfying

$$
\alpha_{P} \phi=\phi \alpha_{Q}
$$

By (3) of the theorem, $\phi$ can be defined as $\phi(x)=T^{-1} x, \forall x \in$ $\operatorname{sl}(n+1, \mathbb{C})$. Then $\forall x \in \operatorname{sl}(n+1, \mathbb{C}),(20)$ is equivalent to

$$
\begin{aligned}
\alpha_{P} \phi(x) & =\phi \alpha_{\mathrm{Q}}(x) \\
& \Longrightarrow P^{-1} T^{-1} x P=T^{-1} Q^{-1} x Q \\
& \Longrightarrow T^{-1} P^{-1} x P=T^{-1} Q^{-1} x Q \\
& \Longrightarrow P^{-1} x P=Q^{-1} x Q \\
& \Longrightarrow Q P^{-1} x=x Q P^{-1} .
\end{aligned}
$$

By Schur's lemma, we have $Q P^{-1}=k E \Rightarrow Q=k P$, for some $k \in \mathbb{C}$.

(7) According to (6) of the theorem,

$$
\begin{aligned}
& \left(s l(n+1, \mathbb{C}),[\cdot, \cdot]_{\alpha_{k_{1} P}}, \alpha_{k_{1} P}\right) \cong\left(s l(n \widehat{+1, \mathbb{C}})_{T_{1}},[\cdot, \cdot]_{T_{1} \alpha_{P}}, \alpha_{P}\right) \\
& \left(s l(n+1, \mathbb{C}),[\cdot, \cdot]_{\alpha_{k_{2} Q}}, \alpha_{k_{2} Q}\right) \cong\left(\operatorname{sl}(n \widehat{+1, \mathbb{C}})_{T_{2}},[\cdot, \cdot]_{T_{2} \alpha_{Q}}, \alpha_{Q}\right) .
\end{aligned}
$$

Then

$$
\begin{aligned}
& \left(s l(n+\overline{1, \mathbb{C}})_{T_{1}},[\cdot, \cdot]_{T_{1} \alpha_{P}}, \alpha_{P}\right) \\
& \cong\left(\operatorname{sl}(n \widehat{+1, \mathbb{C}})_{T_{2}},[\cdot, \cdot]_{T_{2} \alpha_{Q}}, \alpha_{Q}\right) \\
& \quad \Leftrightarrow\left(s l(n+1, \mathbb{C}),[\cdot, \cdot]_{\alpha_{k_{1} P}}, \alpha_{k_{1} P}\right) \\
& \cong\left(s l(n+1, \mathbb{C}),[\cdot, \cdot]_{\alpha_{k_{2} Q}}, \alpha_{k_{2} Q}\right) .
\end{aligned}
$$

Suppose $\phi$ is an isomorphism of $\left(s l(n+1, \mathbb{C}),[\cdot, \cdot]_{\alpha_{k_{1} P}}, \alpha_{k_{1} P}\right)$ and $\left(s l(n+1, \mathbb{C}),[\cdot, \cdot]_{\alpha_{k_{2} Q}}, \alpha_{k_{2} Q}\right)$, and then $\phi$ is an automorphism of $(\operatorname{sl}(n+1, \mathbb{C}),[\cdot, \cdot])$ satisfying

$$
\alpha_{k_{2} Q} \phi=\phi \alpha_{k_{1} P}
$$


By Lie theory $\phi$ can be defined by $\phi(x)=R^{-1} x R, \forall x \in \operatorname{sl}(n+$ $1, \mathbb{C}), R \in g L(V)$. Then (24) is equivalent to

$$
\begin{aligned}
& \alpha_{k_{2} Q} \phi(x)=\phi \alpha_{k_{1} P}(x) \\
& \Longrightarrow\left(k_{2} Q\right)^{-1} R^{-1} x R\left(k_{2} Q\right) \\
&=R^{-1}\left(k_{1} P\right)^{-1} x\left(k_{1} P\right) R \\
& \Longrightarrow P R Q^{-1} R^{-1} x=x P R Q^{-1} R^{-1}, \\
& \forall x \in s l(n+1, \mathbb{C}) .
\end{aligned}
$$

By Schur's lemma we have

$$
P R Q^{-1} R^{-1}=k E \Longrightarrow P R=k R Q \Longrightarrow P=k R Q R^{-1} .
$$

That is, $P$ and $k Q$ are conjugate (for some $k \in \mathbb{C}$ ).

Theorem 11. Let $V$ be a $2 n$-dimensional vector space with a nondegenerate skew symmetric bilinear form $f: V \times V \rightarrow \mathbb{C}$. A subspace sp $\left(\widehat{2 n, \mathbb{C})_{T}}\right.$ of $g l(V)$ is set

$$
\begin{aligned}
s p(\widehat{2 n, \mathbb{C}})_{T} & =\{\widehat{x} \in g l(V) \mid f(\widehat{x} T(v), w)=-f(v, \widehat{x} T(w))\} \\
& =\left\{\widehat{x} \in g l(V) \mid s \widehat{x} T=-T^{t} \widehat{x}^{t} s, s=\left(\begin{array}{cc}
0 & I_{l} \\
-I_{l} & 0
\end{array}\right)\right\},
\end{aligned}
$$

$\forall v, w \in V$.

(1) On $s p(\widehat{2 n, \mathbb{C}})_{T}$, define a bracket $[\cdot, \cdot]_{T}$ by $[\widehat{x}, \widehat{y}]_{T}=$ $\hat{x} T \hat{y}-\hat{y} T \hat{x}, \forall \hat{x}, \hat{y} \in \operatorname{sp}(\widehat{2 n, \mathbb{C}})_{T}$. Then $\left(\operatorname{sp}(2 n, \mathbb{C})_{T},[\cdot, \cdot]_{T}\right)$ is a II type Iso-Lie algebra (we call it an $C_{n}$-Iso-Lie algebra). Define an invertible linear map $\alpha_{P}$ by $\alpha_{P}(\widehat{x})=P^{-1} \widehat{x} P, \forall \widehat{x} \in$ $s p(\widehat{2 n, \mathbb{C}})_{T}$, where $P \in g L(V)$ satisfying $P T=T P ; P^{t} s P=$ s. Then $\alpha_{P}$ is an Iso-automorphism of $\left(\operatorname{sp}(\widehat{2 n, \mathbb{C}})_{T},[\cdot, \cdot]_{T}\right)$. Furthermore,

$$
\begin{aligned}
& S P(\widehat{2 n, \mathbb{C}})_{T} \\
& \quad=\left\{P \in g L(V) \mid P T=T P, P^{t} s P=s, s=\left(\begin{array}{cc}
0 & I_{l} \\
-I_{l} & 0
\end{array}\right)\right\}
\end{aligned}
$$

is a group (we call it the Iso-symplectic group).

(2) Let $s p(2 n, \mathbb{C})$ be a conventional symplectic Lie algebra. Define an invertible linear map $\phi$ by $\phi(x)=x T^{-1}, \forall x \in$ $s p(2 n, \mathbb{C})$. Then $\phi$ is an isotopy from $(s p(2 n, \mathbb{C}),[\cdot, \cdot])$ to $\left(\operatorname{sp}(\widehat{2 n, \mathbb{C}})_{T},[\cdot, \cdot]_{T}\right)$. Furthermore, $\left(\operatorname{sp}(\widehat{2 n, \mathbb{C}})_{T},[\cdot, \cdot]_{T}\right)$ is simple.

(3) Let $\left(\operatorname{sp}(\overline{2 n, \mathbb{C}})_{T_{1}},[\cdot, \cdot]_{T_{1}}\right)$ and $\left(\operatorname{sp}(\overline{2 n, \mathbb{C}})_{T_{2}},[\cdot, \cdot]_{T_{2}}\right)$ be $C_{n}$-Iso-Lie algebras, and then they are isotopic.

(4) On sp $(\widehat{2 n, \mathbb{C}})_{T}$, define a new bracket $[\cdot, \cdot]_{T \alpha_{P}}$ by

$$
\begin{array}{r}
\left([\hat{x}, \widehat{y}]_{T \alpha_{P}}\right)=\alpha_{P}\left([\hat{x}, \hat{y}]_{T}\right)=P^{-1}(\widehat{x} T \hat{y}-\widehat{y} T \widehat{x}) P, \\
\forall \hat{x}, \widehat{y} \in s p\left(\widehat{2 n, \mathbb{C})_{T}} .\right.
\end{array}
$$

Then $\left(\operatorname{sp}(\widehat{2 n, \mathbb{C}})_{T},[\cdot, \cdot]_{T \alpha_{P}}, \alpha_{P}\right)$ is a simple Iso-Hom-Lie algebra (we call it an $C_{n}$-Iso-Hom-Lie algebra). Define an invertible linear map $\psi$ of $\operatorname{sp}(\widehat{2 n, \mathbb{C}})_{T}$ by $\psi(\hat{x})=Q^{-1} \hat{x} Q, \forall \hat{x} \in \operatorname{sp}(\widehat{2 n, \mathbb{C}})_{T}$, where $Q \in S P(\widehat{2 n, \mathbb{C}})_{T}$ satisfies $P Q=k Q P$, for some $k \in \mathbb{C}$; then $\psi$ is an Iso-automorphism of $\left(\operatorname{sp}(\widehat{2 n, \mathbb{C}})_{T},[\cdot, \cdot]_{T \alpha_{P}}, \alpha_{P}\right)$.

(5) Let $\left(\operatorname{sp}(2 n, \mathbb{C}),[\cdot, \cdot]_{\alpha_{\mathrm{Q}}}, \alpha_{\mathrm{Q}}\right)$ be a Hom-Lie algebra with

$$
\begin{gathered}
\alpha_{\mathrm{Q}}(x)=Q^{-1} x Q ;[x, y]_{\alpha_{Q}}=Q^{-1}(x y-y x) Q, \\
\forall x, y \in \operatorname{sp}(2 n, \mathbb{C}), \quad Q \in S P(2 n, \mathbb{C}) .
\end{gathered}
$$

If $Q= \pm P$ is satisfied, then $\left(\operatorname{sp}(2 n, \mathbb{C}),[\cdot, \cdot]_{\alpha_{Q}}, \alpha_{Q}\right)$ is isotopic to $\left(\operatorname{sp}(\widehat{2 n, \mathbb{C}})_{T},[\cdot, \cdot]_{T \alpha_{P}}, \alpha_{P}\right)$.

(6) Let $\left(\operatorname{sp}(\widehat{2 n, \mathbb{C}})_{T_{1}},[\cdot, \cdot]_{T_{1} \alpha_{P}}, \alpha_{P}\right)$ and $\left(s p(\widehat{2 n, \mathbb{C}})_{T_{2}}\right.$, $\left.[\cdot, \cdot]_{T_{2} \alpha_{Q}}, \alpha_{\mathrm{Q}}\right)$ be $C_{n}$-Iso-Hom-Lie algebras. They are isotopic if and only if $P$ and $k Q$ are conjugate (for some $k \in \mathbb{C}$ ).

Proof. (1) Let $\forall \hat{x}, \widehat{y} \in \operatorname{sp}(\widehat{2 n, \mathbb{C}})_{T}$,

$$
\begin{aligned}
s[\widehat{x}, \widehat{y}]_{T} T & =s(\widehat{x} T \hat{y}-\widehat{y} T \widehat{x}) T=-T^{t} \widehat{x}^{t} s \widehat{y} T+T^{t} \widehat{y}^{t} s \widehat{x} T \\
& =T^{t} \widehat{x}^{t} T^{t} \widehat{y}^{t} s-T^{t} \widehat{y}^{t} T^{t} \widehat{x}^{t} s=-T^{t}[\widehat{x}, \widehat{y}]_{T}^{t} s .
\end{aligned}
$$

So $[\widehat{x}, \widehat{y}]_{T} \in s p(\widehat{2 n, \mathbb{C}})_{T}$. The Jacobi identity can be checked directly. Hence $\left(s p(\widehat{2 n, \mathbb{C}})_{T},[\cdot, \cdot]_{T}\right)$ is an II-type Iso-Lie algebra.

On $s p(\widehat{2 n, \mathbb{C}})_{T}$, define an invertible linear map $\alpha_{P}$ by $\alpha_{P}(\widehat{x})=P^{-1} \widehat{x} P, \forall \widehat{x} \in s p(\widehat{2 n, \mathbb{C}})_{T}$, where $P \in g L(V)$ satisfying $P T=T P ; P^{t} s P=s$, we have

$$
\begin{aligned}
s \widehat{x} T & =-T^{t} \widehat{x}^{t} s \\
& \Longrightarrow P^{t} s P P^{-1} \widehat{x} P P^{-1} T P \\
& =-P^{t} T^{t}\left(P^{-1}\right)^{t} P^{t} \widehat{x}^{t}\left(P^{-1}\right)^{t} P^{t} s P \\
& \Longrightarrow s \alpha_{P}(\widehat{x}) T=-T^{t} \alpha_{P}\left((\widehat{x})^{t}\right) s .
\end{aligned}
$$

So $\alpha_{P}\left(s p(\widehat{2 n, \mathbb{C}})_{T}\right) \subseteq s p(\widehat{2 n, \mathbb{C}})_{T} . \forall \hat{x}, \widehat{y} \in s p(\widehat{2 n, \mathbb{C}})_{T}$,

$$
\begin{aligned}
\alpha_{P}\left([\hat{x}, \widehat{y}]_{T}\right) & =P^{-1}(\widehat{x} T \widehat{y}-\widehat{y} T \widehat{x}) P \\
& =P^{-1} \widehat{x} P P^{-1} T P P^{-1} \widehat{y} P-P^{-1} \widehat{y} P P^{-1} T P P^{-1} \widehat{x} P \\
& =P^{-1} \widehat{x} P T P^{-1} \widehat{y} P-P^{-1} \widehat{y} P T P^{-1} \widehat{x} P \\
& =\left[\alpha_{P}(\widehat{x}), \alpha_{P}(\widehat{y})\right]_{T} .
\end{aligned}
$$

Therefore, $\alpha_{P}$ is an Iso-automorphism of $\left(\operatorname{sp}(\widehat{2 n, \mathbb{C}})_{T},[\cdot, \cdot]_{T}\right)$. Let $\forall P_{1}, P_{2} \in S P(\widehat{2 n, \mathbb{C}})_{T}$,

$$
\begin{gathered}
P_{1} P_{2} T=P_{1} T P_{2}=T P_{1} P_{2} \\
\left(P_{1} P_{2}\right)^{t} s\left(P_{1} P_{2}\right)=P_{2}^{t} P_{1}^{t} s P_{1} P_{2}=P_{2}^{t} s P_{2}=s
\end{gathered}
$$

Hence $P_{1} P_{2} \in S P(\widehat{2 n, \mathbb{C}})_{T}$. It is obvious that $E$ is a unit of $S P(\widehat{2 n, \mathbb{C}})_{T}$ and for every element $P \in S P(\widehat{2 n, \mathbb{C}})_{T}$, there is an invertible element $P^{-1} \in S P(\widehat{2 n, \mathbb{C}})_{T}$. We have that $\operatorname{SP}(\widehat{2 n, \mathbb{C}})_{T}$ is a group. 
(2) Because $s \phi(x) T=s x T^{-1} T=s x=-x^{t} s=$ $-T^{t} \phi(x)^{t} s, \forall x \in \operatorname{sp}(2 n, \mathbb{C})$

$$
\begin{array}{r}
\phi(s p(2 n, \mathbb{C})) \subseteq s p(\widehat{2 n, \mathbb{C}})_{T}, \quad \phi([x, y])=[\phi(x), \phi(y)]_{T}, \\
\forall x, y \in s p(2 n, \mathbb{C})
\end{array}
$$

can be checked as in (3) of Theorem 10. Therefore, $\phi$ is an isotopy from $(s p(2 n, \mathbb{C}),[\cdot, \cdot])$ to $\left(s p(\widehat{2 n, \mathbb{C}})_{T},[\cdot, \cdot]_{T}\right)$. $\left(s p(\widehat{2 n, \mathbb{C}})_{T},[\cdot, \cdot]_{T}\right)$ is simple and can be proved as (3) of Theorem 10.

(3) Define an invertible linear map $\phi$ by $\phi(\widehat{x})=$ $\widehat{x} T_{1} T_{2}^{-1}, \forall \widehat{x} \in \operatorname{sp}(\widehat{2 n, \mathbb{C}})_{T_{1}}$. Then

$$
\begin{aligned}
& \phi(\widehat{x}) \in s p(\widehat{2 n, \mathbb{C}})_{T_{2}}, \quad \phi\left([\hat{x}, \widehat{y}]_{T_{1}}\right)=[\phi(\widehat{x}), \phi(\widehat{y})]_{T_{2}}, \\
& \forall \hat{x}, \widehat{y} \in s p(\widehat{2 n, \mathbb{C}})_{T_{1}}
\end{aligned}
$$

can be proved as (2) of the proof. Therefore, $\phi$ is an isotopy from $\left(s p(\widehat{2 n, \mathbb{C}})_{T_{1}},[\cdot, \cdot]_{T_{1}}\right)$ to $\left(s p(\widehat{2 n, \mathbb{C}})_{T_{2}},[\cdot, \cdot]_{T_{2}}\right)$.

(4) $\left(\operatorname{sp}(\widehat{2 n, \mathbb{C}})_{T},[\cdot, \cdot]_{T \alpha_{P}}, \alpha_{P}\right)$ is a simple Iso-Hom-Lie algebra and can be got from (1) and (3) of Theorem, Lemma 3, and Proposition 7 directly. Suppose $\psi$ is an Iso-automorphism of $\left(s p(\widehat{2 n, \mathbb{C}})_{T},[\cdot, \cdot]_{T \alpha_{P}}, \alpha_{P}\right)$, and according to Lemma $6, \psi$ is an Iso-automorphism of the Hom-Lie admissible algebra $\left(s p(\widehat{2 n, \mathbb{C}})_{T},[\cdot, \cdot]_{T}\right)$ satisfying

$$
\alpha_{P} \psi=\psi \alpha_{P}
$$

By (1) of the theorem, $\psi$ can be defined by $\psi(\hat{x})=$ $Q^{-1} \widehat{x} Q, \forall \widehat{x} \in \operatorname{sp}(\widehat{2 n, \mathbb{C}})_{T}$, where $Q \in S P(\widehat{2 n, \mathbb{C}})_{T}$. So (37) is equivalent to

$$
\begin{gathered}
\alpha_{P} \psi(\widehat{x})=\psi \alpha_{P}(\widehat{x})=P^{-1} Q^{-1} \widehat{x} Q P=Q^{-1} P^{-1} \widehat{x} P Q \\
\Longrightarrow P Q P^{-1} Q^{-1} \widehat{x}=\widehat{x} P Q P^{-1} Q^{-1} \\
\forall \widehat{x} \in \operatorname{sp}(\widehat{2 n, \mathbb{C}})_{T} .
\end{gathered}
$$

By (2) of the proof, $\exists x^{\prime} \in \operatorname{sp}(2 n, \mathbb{C})$ such that $\widehat{x}=x^{\prime} T^{-1}$. Then (38) is equivalent to

$$
\begin{aligned}
P Q P^{-1} Q^{-1} x^{\prime} T^{-1} & =x^{\prime} T^{-1} P Q P^{-1} Q^{-1} \\
& =x^{\prime} P Q P^{-1} Q^{-1} T^{-1} \\
& \Longrightarrow P Q P^{-1} Q^{-1} x^{\prime} \\
& =x^{\prime} P Q P^{-1} Q^{-1}
\end{aligned}
$$

By the arbitrariness of $\widehat{x}$, we have for all $x^{\prime} \in s p(2 n, \mathbb{C}),(39)$ is established. According to Schur's lemma, we have $P Q=k Q P$, for some $k \in \mathbb{C}$.

(5) Suppose $\phi$ is an isotopy from $\left(\operatorname{sp}(2 n, \mathbb{C}),[\cdot, \cdot]_{\alpha_{Q}}, \alpha_{Q}\right)$ to $\left(\operatorname{sp}(\widehat{2 n, \mathbb{C}})_{T},[\cdot, \cdot]_{T \alpha_{P}}, \alpha_{P}\right)$. According to Lemma $5, \phi$ is an isotopy of their Hom-Lie admissible algebras satisfying

$$
\alpha_{P} \phi=\phi \alpha_{\mathrm{Q}}
$$

By (2) of theorem, $\phi$ can be defined by $\phi(x)=x T^{-1}, \forall x \in$ $s p(2 n, \mathbb{C})$. Equation(40) is equivalent to

$$
\begin{aligned}
\alpha_{P} \phi(x)= & \phi \alpha_{\mathrm{Q}}(x) \\
& \Longrightarrow P^{-1} x T^{-1} P=Q^{-1} x Q T^{-1} \\
\Longrightarrow & P^{-1} x P T^{-1}=Q^{-1} x Q T^{-1} \\
\Longrightarrow & P^{-1} x P=Q^{-1} x Q \\
\Longrightarrow & Q P^{-1} x=x Q P^{-1}, \\
& \forall x \in \operatorname{sp}(2 n, \mathbb{C}) .
\end{aligned}
$$

By Schur's lemma we have $Q=k P$, for some $k \in \mathbb{C}$. According to (4) of the theorem, $P \in S p(\widehat{2 n, \mathbb{C}})_{T}$, so $P^{t} s P=s$. Because $Q \in S P(2 n, \mathbb{C})$, then $Q^{t} s Q=s \Rightarrow k P^{t} s k P=k^{2} P^{t} s P=k^{2} s=$ $s \Rightarrow k= \pm 1$. We have the conclusion.

(6) The same reason as (7) of Theorem 10, $\left(s p(\overline{2 n, \mathbb{C}})_{T_{1}},[\cdot, \cdot]_{T_{1} \alpha_{P}}, \alpha_{P}\right) \quad$ and $\quad\left(s p(\overline{2 n, \mathbb{C}})_{T_{2}},[\cdot, \cdot]_{T_{2} \alpha_{Q}}, \alpha_{Q}\right)$ are isotopic if and only if $\left(\operatorname{sp}(2 n, \mathbb{C}),[\cdot, \cdot]_{\alpha_{ \pm P}}, \alpha_{ \pm P}\right) \cong$ $\left(\operatorname{sp}(2 n, \mathbb{C}),[\cdot, \cdot]_{\alpha_{ \pm Q}}, \alpha_{ \pm Q}\right)$. Suppose $\psi$ is an isomorphism from $\left(s p(2 n, \mathbb{C}),[\cdot, \cdot]_{\alpha_{ \pm P}}, \alpha_{ \pm P}\right)$ to $\left(s p(2 n, \mathbb{C}),[\cdot, \cdot]_{\alpha_{ \pm Q}}, \alpha_{ \pm Q}\right)$, then $\psi$ is an automorphism of $(\operatorname{sp}(2 n, \mathbb{C}),[\cdot, \cdot])$ satisfying

$$
\alpha_{ \pm Q} \psi=\psi \alpha_{ \pm P}
$$

By Lie theory we know $\psi$ can be defined by $\psi(x)=$ $R^{-1} x R, \forall x \in \operatorname{sp}(2 n, \mathbb{C})$, where $R \in S P(2 n, \mathbb{C})$ and $(42)$ is equivalent to

$$
\begin{aligned}
\alpha_{ \pm Q} \psi(x) & =\psi \alpha_{ \pm P}(x) \\
& \Longrightarrow( \pm Q)^{-1} R^{-1} x R( \pm Q) \\
& =R^{-1}( \pm P)^{-1} x( \pm P) R \\
& \Longrightarrow P R Q^{-1} R^{-1} x=x P R Q^{-1} R^{-1}, \\
& \forall x \in \operatorname{sp}(2 n, \mathbb{C}) .
\end{aligned}
$$

By Schur's lemma we have $P R Q^{-1} R^{-1}=k E \Rightarrow R^{-1} P R=k Q$. That is, $P$ is conjugate with $k Q$ (for some $k \in \mathbb{C}$ ).

Theorem 12. Let $V$ be an n-dimensional vector space with a nondegenerate symmetric bilinear form $f: V \times V \rightarrow \mathbb{C}$. Define a subspace so $(\widehat{n, \mathbb{C}})_{T}$ of $g l(V)$ by 
$\operatorname{so}(\widehat{n, \mathbb{C}})_{T}=\{\widehat{x} \in g l(V) \mid f(\widehat{x} T(v), w)=-f(v, \widehat{x} T(w))\}=\left\{\hat{x} \in g l(V) \mid s \widehat{x} T=-T^{t} \widehat{x}^{t} s, s=\left\{\begin{array}{ll}\left(\begin{array}{ccc}1 & 0 & 0 \\ 0 & 0 & I_{l} \\ 0 & I_{l} & 0\end{array}\right), & n=2 l+1 \\ \left(\begin{array}{cc}0 & I_{l} \\ I_{l} & 0\end{array}\right), & n=2 l\end{array}\right\}\right.$.

(1) On so( $(\widehat{n, \mathbb{C}})_{T}$ define a bracket $[\cdot, \cdot]_{T}$ by $[\widehat{x}, \widehat{y}]_{T}=$ $\widehat{x} T \hat{y}-\hat{y} T \hat{x}, \forall \hat{x}, \hat{y} \in \operatorname{so}(\widehat{n, \mathbb{C}})_{T}$, and then $\left(\operatorname{so}(\widehat{n, \mathbb{C}})_{T},[\cdot, \cdot]_{T}\right)$ is an II type $B_{l}(n=2 l+1)$ or $D_{l}(n=2 l)$ Iso-Lie algebra.
Define an invertible linear map $\alpha_{P}$ by $\alpha_{P}(\widehat{x})=P^{-1} \widehat{x} P$, $\forall \hat{x} \in \operatorname{so}(\widehat{n, \mathbb{C}})_{T}$, where $P \in g L(V)$ satisfying $P T=T P, P^{t} s P=$ s. Then $\alpha_{P}$ is an Iso-automorphism of $\left(\right.$ so $\left.(\widehat{n, \mathbb{C}})_{T},[\cdot, \cdot]_{T}\right)$. Furthermore,

$$
S O(\widehat{n, \mathbb{C}})_{T}=\left\{P \in g L(V) \mid P T=T P, P^{t} s P=s, s=\left\{\begin{array}{ccc}
\left(\begin{array}{ccc}
1 & 0 & 0 \\
0 & 0 & I_{l} \\
0 & I_{l} & 0
\end{array}\right), & n=2 l+1 \\
\left(\begin{array}{cc}
0 & I_{l} \\
I_{l} & 0
\end{array}\right), & n=2 l
\end{array}\right\}\right.
$$

is a group (we call it the Iso-orthogonal group).

(2) Let $(\operatorname{so}(n, \mathbb{C}),[\cdot, \cdot])$ be a conventional orthogonal Lie algebra. On so $(n, \mathbb{C})$, define an invertible linear map $\phi$ by $\phi(x)=x T^{-1}, \forall x \in \operatorname{so}(n, \mathbb{C})$. Then $\phi$ is an isotopy from the conventional Lie algebra $(\operatorname{so}(n, \mathbb{C}),[\cdot, \cdot])$ to $\left(\operatorname{so}(\widehat{n, \mathbb{C}})_{T},[\cdot, \cdot]_{T}\right)$. Furthermore $\left(\operatorname{so}(\widehat{n, \mathbb{C}})_{T},[\cdot, \cdot]_{T}\right)$ is simple.

(3) Let $\left(\operatorname{so}(\widehat{n, \mathbb{C}})_{T_{1}},[\cdot, \cdot]_{T_{1}}\right)$ and $\left(\operatorname{so}(\widehat{n, \mathbb{C}})_{T_{2}},[\cdot, \cdot]_{T_{2}}\right)$ be $B_{l}$ (or $D_{l}$ )-Iso-Lie algebras, and then they are isotopic.

(4) On so $(\widehat{n, \mathbb{C}})_{T}$, define a new bracket $[\cdot, \cdot]_{T \alpha_{P}}$ by

$$
\begin{aligned}
\left([\hat{x}, \hat{y}]_{T \alpha_{P}}\right) & =\alpha_{P}\left([\hat{x}, \widehat{y}]_{T}\right) \\
& =P^{-1}(\widehat{x} T \hat{y}-\widehat{y} T \hat{x}) P, \quad \forall \hat{x}, \hat{y} \in \operatorname{so}(\widehat{n, \mathbb{C}})_{T} .
\end{aligned}
$$

Then $\left(\operatorname{so}(\widehat{n, \mathbb{C}})_{T},[\cdot, \cdot]_{T \alpha_{P}}, \alpha_{P}\right)$ is a simple Iso-Hom-Lie algebra (we call it a $B_{l}$ or $D_{l}$-Iso-Hom-Lie algebra). On so $(\widehat{n, \mathbb{C}})_{T}$, define an invertible linear map $\psi$ by $\psi(\widehat{x})=Q^{-1} \widehat{x} Q, \forall \widehat{x} \in$ so $(\widehat{n, \mathbb{C}})_{T}$, where $Q \in S O(\widehat{n, \mathbb{C}})_{T}$ satisfies $P Q=k Q P$, for some $k \in \mathbb{C}$, and then $\psi$ is an Iso-automorphism of $\left(\operatorname{so}(\widehat{n, \mathbb{C}})_{T},[\cdot, \cdot]_{T \alpha_{P}}, \alpha_{P}\right)$.

(5) Let $\left(\operatorname{so}(n, \mathbb{C}),[\cdot, \cdot]_{\alpha_{Q}}, \alpha_{\mathrm{Q}}\right)$ be a Hom-Lie algebra with

$$
\begin{gathered}
\alpha_{\mathrm{Q}}(x)=Q^{-1} x \mathrm{Q} ; \quad[x, y]_{\alpha_{\mathrm{Q}}}=Q^{-1}(x y-y x) \mathrm{Q}, \\
\forall x, y \in \text { so }(n, \mathbb{C}), Q \in S O(n, \mathbb{C}) .
\end{gathered}
$$

If $Q= \pm P$ is satisfied, then $\left(\operatorname{so}(n, \mathbb{C}),[\cdot, \cdot]_{\alpha_{Q}}, \alpha_{Q}\right)$ is isotopic to $\left(\operatorname{so}(\widehat{n, \mathbb{C}})_{T},[\cdot, \cdot]_{T \alpha_{P}}, \alpha_{P}\right)$.
(6) Let $\left(\operatorname{so}(\widehat{n, \mathbb{C}})_{T_{1}},[\cdot, \cdot]_{T_{1} \alpha_{P}}, \alpha_{P}\right)$ and $\left(\operatorname{so}(\widehat{n, \mathbb{C}})_{T_{2}}\right.$, $\left.[\cdot, \cdot]_{T_{2} \alpha_{Q}}, \alpha_{Q}\right)$ be $B_{l}$ (or $D_{l}$ )-Iso-Hom-Lie algebras. They are isotopic if and only if $P$ and $k Q$ are conjugate (for some $k \in \mathbb{C})$.

\section{Acknowledgment}

This project is partially supported by the National Nature science foundation of China (11071187).

\section{References}

[1] R. M. Santilli, "Addendum to: 'On a possible Lie-admissible covering of the Galilei relativity in Newtonian mechanics for nonconservative and Galilei form-noninvariant systems,"' Hadronic Journal, vol. 1, no. 4, pp. 1279-1342, 1978.

[2] R. M. Santilli, "Invariant Lie-admissible formulation of quantum deformations," Foundations of Physics, vol. 27, no. 8, pp. 1159-1177, 1997.

[3] D. S. Sourlas and G. T. Tsagas, Mathematical Foundations of the Lie-Santilli Theory, "Naukova Dumka”, Kiev, Ukraine, 1993.

[4] J. T. Hartwig, D. Larsson, and S. D. Silvestrov, "Deformations of Lie algebras using $\sigma$-derivations," Journal of Algebra, vol. 295, no. 2, pp. 314-361, 2006.

[5] D. Larsson and S. D. Silvestrov, "Quasi-Lie algebras," Contemporary Mathematics, vol. 391, pp. 241-248, 2005.

[6] D. Larsson and S. D. Silvestrov, "Quasi-hom-Lie algebras, central extensions and 2-cocycle-like identities," Journal of Algebra, vol. 288, no. 2, pp. 321-344, 2005. 
[7] N. Hu, " $q$-Witt algebras, $q$-Lie algebras, $q$-holomorph structure and representations," Algebra Colloquium, vol. 6, no. 1, pp. 51-70, 1999.

[8] Q. Jin and X. Li, "Hom-Lie algebra structures on semi-simple Lie algebras," Journal of Algebra, vol. 319, no. 4, pp. 1398-1408, 2008.

[9] D. Yau, "The Hom-Yang-Baxter equation, Hom-Lie algebras, and quasi-triangular bialgebras," Journal of Physics A, vol. 42, no. 16, pp. 165-202, 2009.

[10] A. Makhlouf and S. D. Silvestrov, "Hom-algebra structures," Journal of Generalized Lie Theory and Applications, vol. 2, no. 2, pp. 51-64, 2008.

[11] J. E. Humphreys, Introduction to Lie Algebras and Representation Theory, Springer, New York, NY, USA, 1972.

[12] N. Jacobson, Lie Algebras, Dover, New York, NY, USA, 1962.

[13] Y. Sheng, "Representations of hom-Lie algebras," Algebras and Representation Theory, vol. 15, no. 6, pp. 1081-1098, 2012.

[14] D. Yau, "Hom-algebras and homology," Journal of Lie Theory, vol. 19, no. 2, pp. 409-421, 2009.

[15] D. Yau, "The Hom-Yang-Baxter equation and Hom-Lie algebras," Journal of Mathematical Physics, vol. 52, no. 5, 2011. 


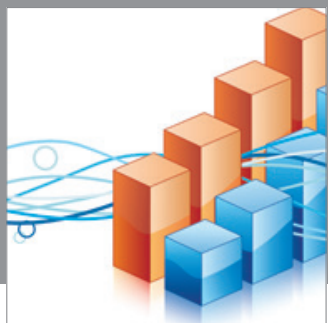

Advances in

Operations Research

mansans

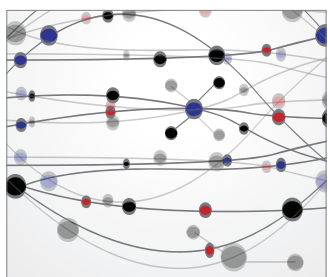

The Scientific World Journal
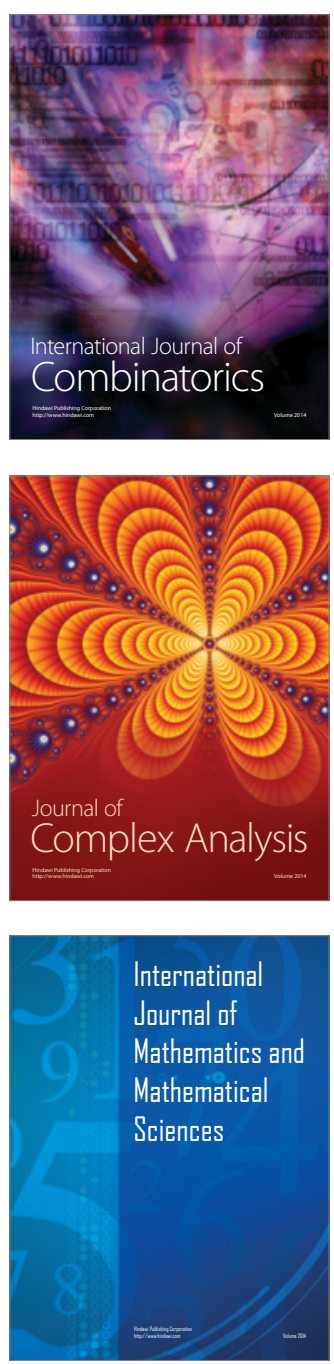
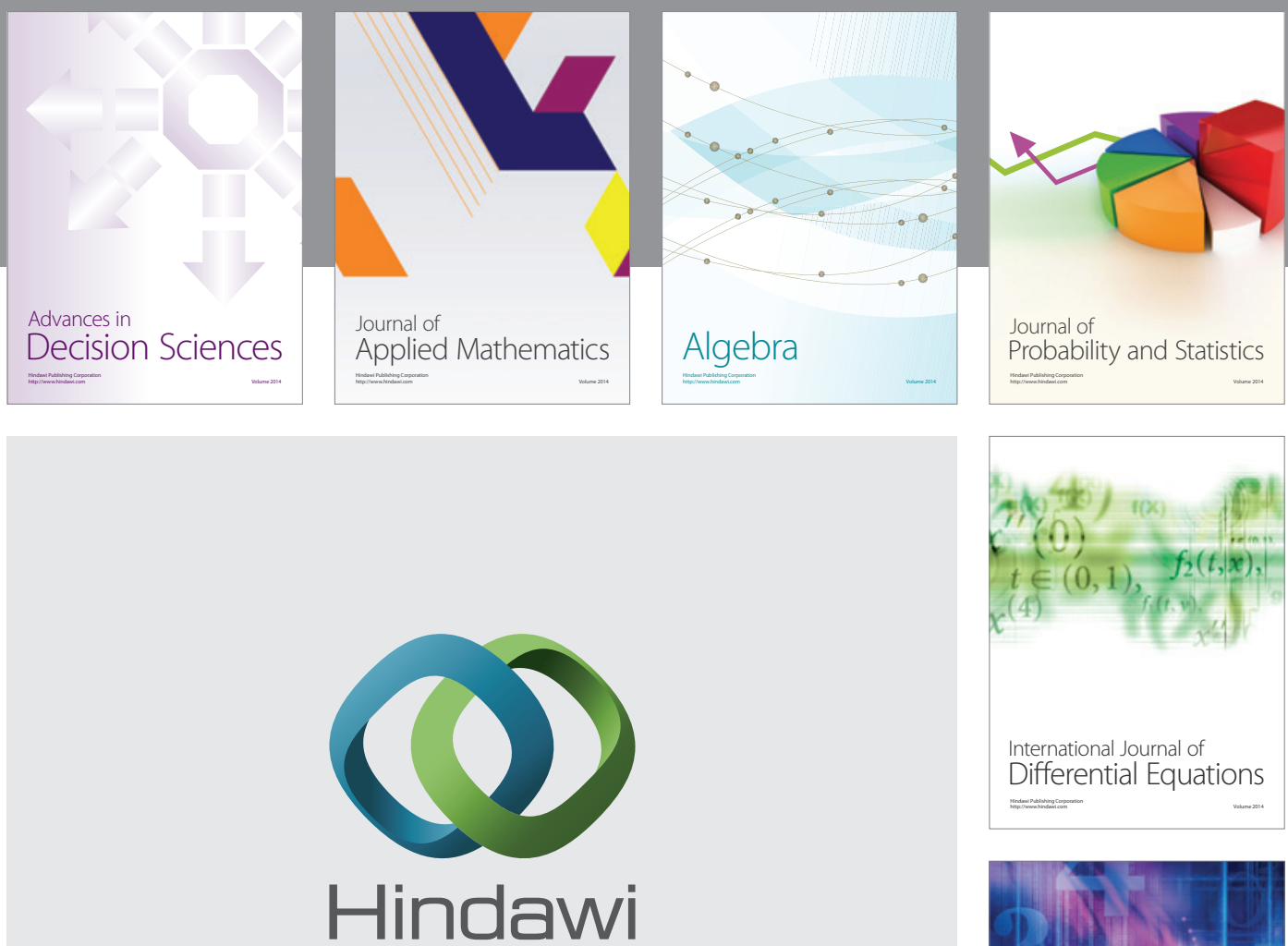

Submit your manuscripts at http://www.hindawi.com
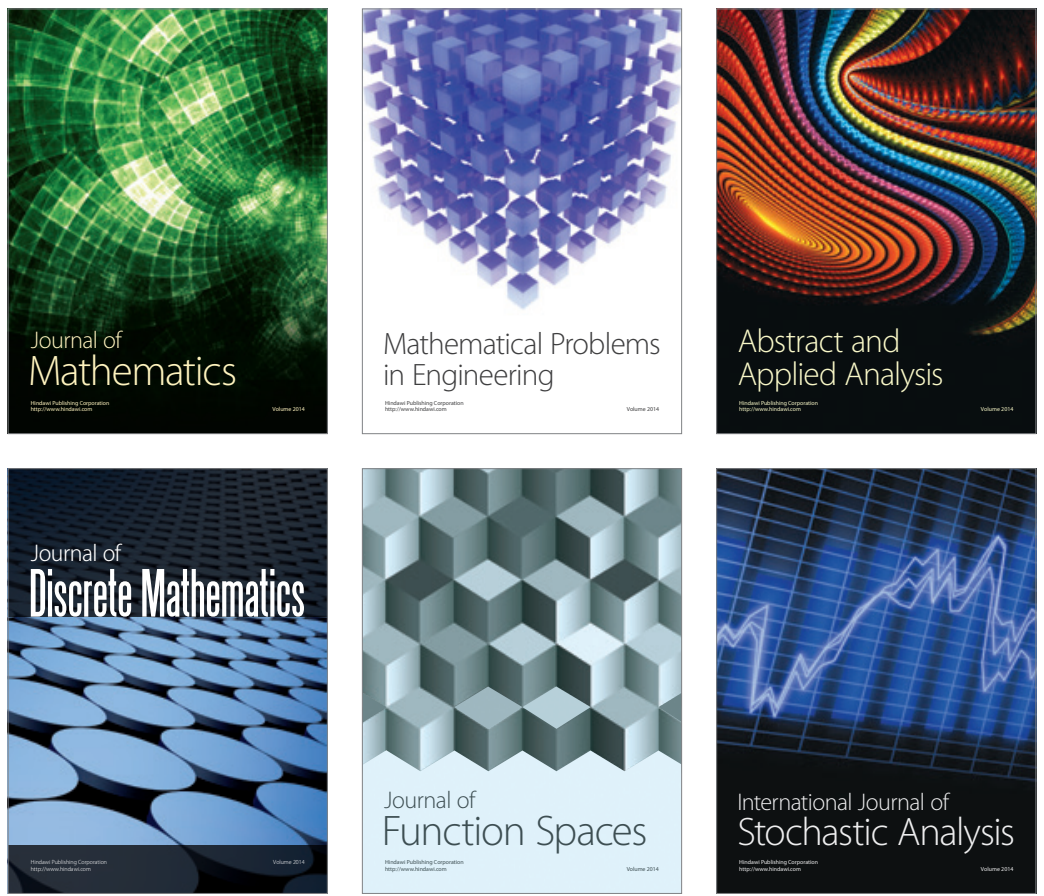

Journal of

Function Spaces

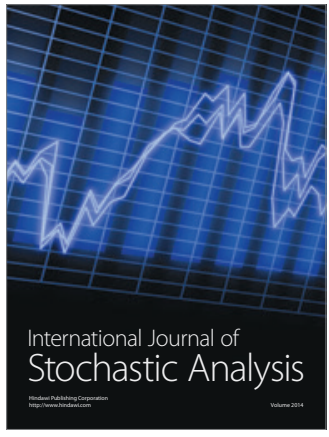

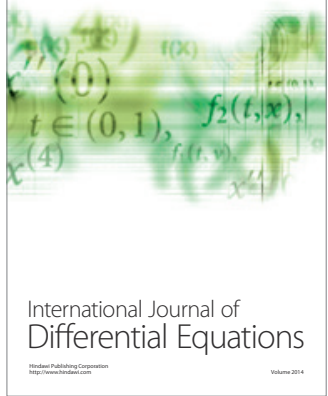
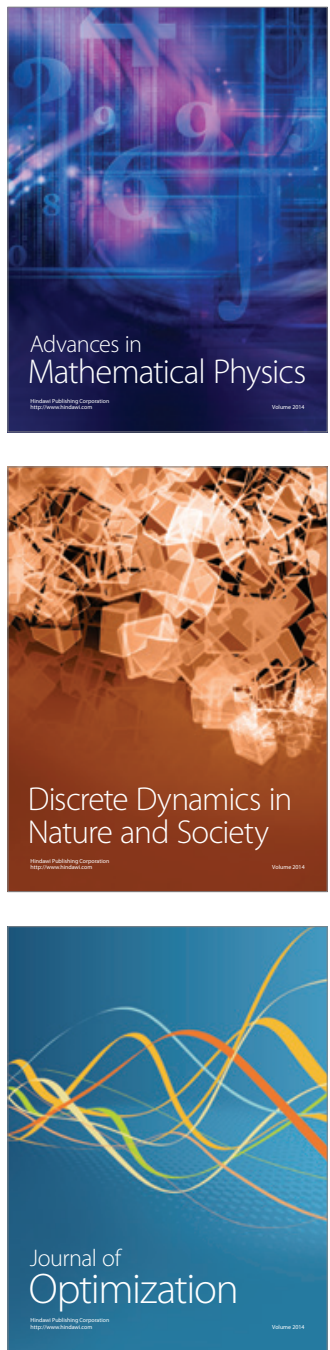